\title{
Probing the Dynamics of Topologically Protected Charged Ferroelectric Domain Walls with the Electron Beam at the Atomic Scale
}

Michele Conroy ${ }^{1}$, Kalani Moore ${ }^{1}$, Eoghan O'Connell $^{1}$, Lewys Jones ${ }^{2}$, Clive Downing ${ }^{3}$, Roger Whamore ${ }^{4}$, Alexei Gruverman ${ }^{5}$, Marty Gregg ${ }^{6}$ and Ursel Bangert ${ }^{1}$

${ }^{1}$ University of Limerick, Limerick, Limerick, Ireland, ${ }^{2}$ Trinity College Dublin, Dublin, Dublin, Ireland, ${ }^{3}$ Advanced Microscopy Laboratory, Centre for Research on Adaptive Nanostructures and Nanodevices (CRANN), Dublin 2, Ireland, Dublin, Dublin, Ireland, ${ }^{4}$ Imperial College London, London, England, United Kingdom, ${ }^{5}$ University of Nebraska Lincoln, Lincoln, Nebraska, United States, ${ }^{6}$ Queen's University Belfast, Belfast, Northern Ireland, United Kingdom

Dynamic charged ferroelectric domain walls (CDWs) overturn the classical idea that our electronic circuits need to consist of fixed components of hardware.[1,2] With their own unique electronic properties and exotic functional behaviours all confined to their nanoscale width, DWs represent a completely new 2D material phase.[3-5] The most exciting aspect of CDWs in single crystals is that they can be easily created, destroyed and moved simply by an applied stimulus. The dynamic nature of CDWs gives them the edge over other novel systems and may lead to them being the next promising disruptive quantum technology. This is an area of research at its very early stages with endless possible applications. However, to harness their true potential there is a great deal of the fundamental physics yet to uncover. As the region of interest (CDW) is atomically thin and dynamic, it is essential for the physical characterisation to be at this scale spatially and time-resolved.

Recently there has been a surge in interest of electron-beam-based atom-by-atom manipulation.[6-9] This presentation will focus on using the applied electric field $\left(\mathrm{E}_{\mathrm{f}}\right)$ of the scanning transmission electron microscope (STEM) probe to move CDWs, and thus investigate their dynamics while imaging at the subatomic scale. As the applied $\mathrm{E}_{\mathrm{f}}$ of an electron probe can be controlled in terms of dose, probe size, direction and speed a diverse set of experiments is possible without complicated sample preparation. Using a segmented STEM detector any changes in deflection and thus the changes in polarisation for each domain and DW, can also be monitored with controlled variants in applied $E_{f}$ conditions. This allows for studying the influence of different domain patterns and DW types (charged and neutral) on the dynamics of the CDWs, Figure 1. By controlling the incoming STEM probe direction, parallel DWs could be moved around to form stable four-fold charged junctions thus switching DWs from a neutral to charged state. Deceasing the dose also reduces the speed of the DW movement and thus the formation of the these junctions can be captured using the HAADF detector at the atomic scale. Then in each frame by quantifying the atomic displacement per unit cell using Atomap,[10] the local polarisation at these topological protected CDWs can also be monitored, Figure 2.

The results of this presentation show how the applied $\mathrm{E}_{\mathrm{f}}$ of the electron probe is a viable in situ electron microscopy technique providing a new platform for understanding the fundamentals physics of topologically protected quantum state dynamics. Previously thought energetically unfavorable charged junctions can be formed when the applied $E_{f}$ can be controlled at these sub-atomic dimensions. The new insights presented here required the resolution allowed by aberration corrected STEM due to the 2D nature of the CDWs, reinforcing the idea that advancements in STEM techniques are essential for the progress of quantum information sciences. 
(a)

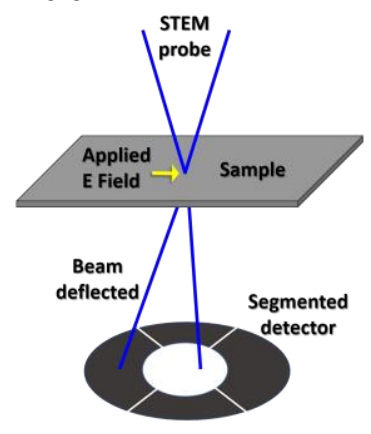

(d)

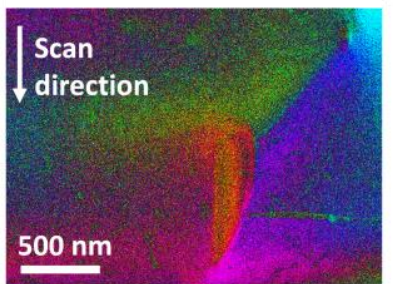

(b)

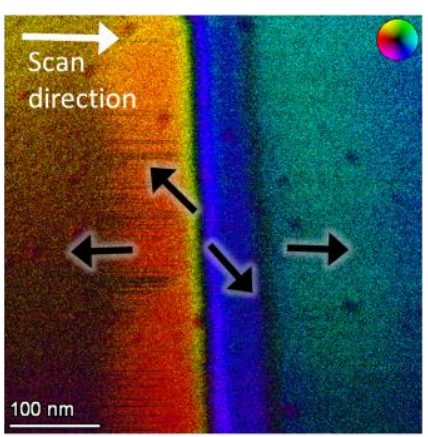

(e)

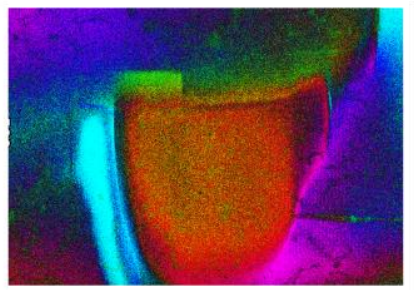

(c)

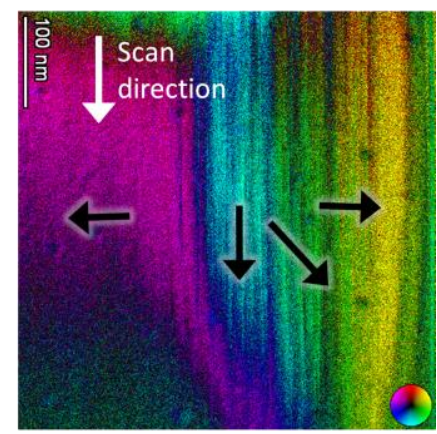

(f)

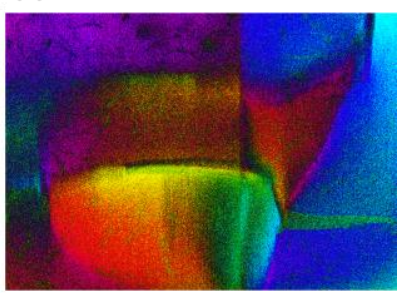

Figure 1. (a) schematic of STEM DPC mapping using a segmented detector, (b) and (c) DPC polarisation mapping of the domains and DWs with changing scan direction of the applied electric field, (d)-(f) domain pattern change with increased dose.

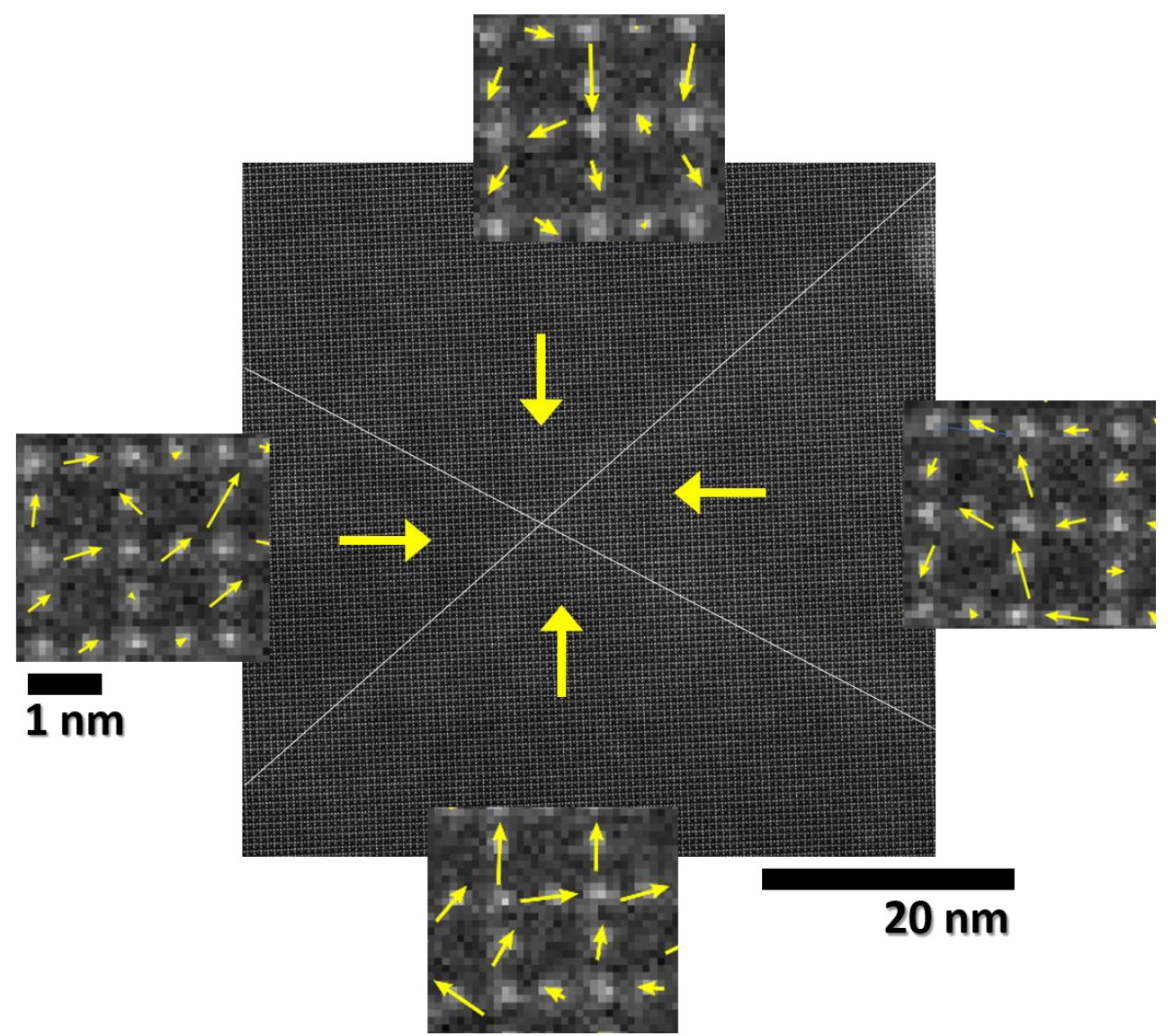

Figure 2. STEM HAADF image of the four fold charged junction formed under the STEM probe. 


\author{
References \\ [1] Catalan, G et al. , Domain Wall Nanoelectronics. Rev. Mod. Phys 84 (2012), p. 119. \\ [2] McQuaid, R et al. , Nature Communications 8 (2017). \\ [3]Jiang, J et al. , Nature Materials 17 (17) (2017), p. 49. \\ [4]McGilly, L. J et al. , Nature Nanotechnology 10 (2015), p. 145. \\ [5]Seidel, J et al. , J. Phys. Chem. Lett 19 (2012), p. 2905. \\ [6] Hage, F. S et al., Advanced Functional Materials 29.52 (2019) 1901819. \\ [7] Kalinin, S V., and Pennycook, S. MRS Bulletin 42.9 (2017) 637-643. \\ [8] Dyck, O, et al. Small 14.38 (2018) 1801771. \\ [9] Maksov, A et al. npj Computational Materials 5.1 (2019) 1-8. \\ [10] Nord, M et al. , Adv Struct Chem Imaging 3 (2017) p. 9.
}

